\title{
ON THE REPRESENTATION OF SIMPLE RIESZ GROUPS.
}

\author{
E. Pardo* \\ Departament de Matemtiques \\ Universitat Autònoma de Barcelona \\ 08193 Bellaterra (Barcelona), Spain \\ e-mail address: epardo@mat.uab.es
}

Dedicat al Francesc $i$ la Montse.

\begin{abstract}
In this paper we answer Open Problem 2 of Goodearl's book on partially ordered abelian groups in the case of partially ordered simple groups. As a consequence, we obtain a version of the Theorem of structure of dimension groups in the case of simple Riesz groups. Also, we give a method for constructing torsion-free strictly perforated simple Riesz groups of rank one, and we see that every dense additive subgroup of $\mathbb{Q}$ can be obtained using this method.
\end{abstract}

\section{Introduction}

In [5], Effros, Handelman and Shen gave one of the most important results in the theory of ordered groups: the Theorem of structure of dimension groups. This Theorem characterizes these groups as direct limits of direct systems of

*Partially supported by DGICYT grants no. PB-93-0900 and PB-95-0626, and by the Comissionat per Universitats i Recerca de la Generalitat de Catalunya. This paper is part of the author's Ph. D. Thesis, written under the supervision of Professor P. Ara. 
simplicial groups (i.e., groups of the form $\mathbb{Z}^{n}$ for some $n \in \mathbb{N}$, endowed with the product ordering). It is closely related to a previous paper [6], where Elliott stated the classification of all AF $C^{*}$-algebras, by using the ordered $K_{0}$ group as an invariant. Thus, a natural question, in order to study the structure of wider classes of ordered abelian groups - as well as for its potential applications to K-Theory - is how far one could go in the way of obtaining (partial) versions of this Theorem in the case of groups satisfying weaker hypotheses. In particular, Goodearl, in Open Problems 1 and 2 of [8], asks what kind of results about dimension groups hold without the unperforation assumption. These questions are related with [7, Open Problem 27], where the question is whether $K_{0}(R)$ is unperforated and torsion-free for every unit-regular ring $R$, and also with the analogous questions for simple $C^{*}$-algebras of real rank zero, which can be found in [3]. These questions have negative answers, as shown by Goodearl in [9] for the case of simple unit-regular rings, and by Blackadar in [2] for the case of simple $C^{*}$-algebras of real rank zero, with the construction of rings of these two classes whose $K_{0}$ group is an interpolation group with torsion. Nevertheless, as far as we know, there are no known examples of Grothendieck groups in those classes that are simultaneously perforated and torsion-free.

In this note, we use the results of [12] about the density of the image of the representation of a pre-ordered abelian group with order-unit into the space of affine and continuous functions on its state space to answer [8, Open Problem $2]$ in the affirmative, as far as simple Riesz groups are concerned, because the answer in the general case is negative, as Dugas shows in [4] by constructing a counterexample. This result allows us to obtain a version of the Theorem of Effros, Handelman and Shen in the case of simple Riesz groups, by using building blocks similar to simplicial groups. Unfortunately, our version only guarantees that this kind of groups are direct limits of some basic building blocks, not all of which are necessarily simple. Also, we give a method for building examples of torsion-free strictly perforated simple Riesz groups, which shows that our generalization is not vacuous (after we constructed our example, K. R. Goodearl informed us of an unpublished example of Lawrence [10] with the same pathology, which is different from ours. Also, in the final revision of this note, the referee communicated us that the existence of an example similar to ours was quoted by Wehrung, without proof, in [13]). In fact we show that every dense additive subgroup of $\mathbb{Q}$ containing $\mathbb{Z}$ can be built in this way.

Throughout this note we will refer to [8] for notations and definitions on partially ordered abelian groups. We recall here some basic definitions about the context of this note. Given an abelian group $G$, a cone of $G$ is an additive submonoid $P$ of $G$ containing zero, and we say that the cone $P$ is strict if $P \cap(-P)=\{0\}$. Thus, a partially ordered abelian group is an abelian group $G$ endowed with a strict cone, called the positive cone of $G$ and denoted by $G^{+}$, whose elements are called the positive elements of $G$. Also, we denote 
$G^{++}=G^{+} \backslash\{0\}$ (so that $\mathbb{Z}^{++}=\mathbb{N}$ ). A partially ordered abelian group $G$ is said to be directed provided that any element can be written as a difference of two positive elements. Given a partially ordered abelian group $G$, an element $u \in G$ is said to be an order-unit provided that $u \in G^{++}$and for each element $x \in G$ there exists $n$ in $\mathbb{N}$ such that $x \leq n u$ (in particular a group with order-unit is directed). A partially ordered abelian group is said to be simple when it is nonzero and every nonzero positive element is an order-unit. A partially ordered abelian group $G$ is said to be (strictly) unperforated provided that for all $x \in G$ and for all $n \in \mathbb{N}$, if $n x \in G^{+}\left(n x \in G^{++}\right)$, then $x \in G^{+}$ $\left(x \in G^{++}\right)$. Finally, a partially ordered abelian group is an interpolation group if for all $x_{1}, x_{2}, y_{1}, y_{2} \in G$ such that $\forall i, j x_{i} \leq y_{j}$, there exists an element $z \in G$ such that $\forall i, j x_{i} \leq z \leq y_{j}$. A Riesz group is a directed interpolation group.

\section{The main result}

In this section we will state a (partial) version of the Theorem of structure of dimension groups in the case of torsion-free simple Riesz groups. The key point to obtain this result is the fact that we can answer in the affirmative [8, Open Problem 2] in the case of simple Riesz groups. This problem asks if the interpolation property of a partially ordered abelian group is preserved if we replace $G^{+}$by: (a) the set of elements $x \in G$ such that $m x \in G^{+}$for some $m \in \mathbb{N}$; (b) the set of elements $x \in G$ such that $2^{n} x \in G^{+}$for some $n \in \mathbb{N}$. Notice that our result proves that a simple Riesz group is, essentially, a simple dimension group in which we have "suppressed" some elements of the positive cone (in particular, as we will see, it could be realized as a direct limit of some special building blocks).

As we noticed above, we will show that the answer to [8, Open Problem 2] is affirmative in the case of simple groups (the non simple case is taken care of in Dugas' example [4]). To do this, we recall a definition that will be necessary. Let $(G, u)$ be a partially ordered abelian group with order-unit, and let $\Phi$ : $G \rightarrow \operatorname{Aff}(S(G, u))$ be the natural affine and continuous representation map (see [8, p. 117]). A partially ordered abelian group with order-unit $(G, u)$ is said to satisfy condition $(D)$ provided that $\Phi\left(G^{+}\right)$is dense in $\operatorname{Aff}(S(G, u))^{+}$(this definition was introduced in [1]). Also recall that, according to $[8$, Proposition 14.3], the state space of a noncyclic simple Riesz group contains no discrete states. Then, by applying [12, Theorem 3.5] we obtain the following result

Lemma 1.1 If $(G, u)$ is a noncyclic, simple Riesz group with order-unit, then $G$ satisfies condition (D).

Definition 1.2 Let $G$ be a partially ordered abelian group. Then, we define the strict unperforated cone of $G$ to be

$$
G^{(c)+}=\left\{x \in G:(\exists n \in \mathbb{N})\left(n x \in G^{++}\right)\right\} \cup\{0\} .
$$


Obviously $G^{(c)+}$ is a strict cone, and we call the canonical strictly unperforated image of $G$, denoted by $G^{(c)}$, the group $G$ endowed with this new cone. Notice that, if $G$ is torsion-free, then $G^{(c)+}=\left\{x \in G:(\exists n \in \mathbb{N})\left(n x \in G^{+}\right)\right\}$, as 0 is not a special case. In order to make clearer the arguments below, we will say that $x \in G$ is a perforated element provided that $x \in G^{(c)+} \backslash G^{+}$.

As a consequence, we can show the desired result:

Theorem 1.3 Let $G$ be a partially ordered abelian group. Then $G^{(c)}$ is strictly unperforated. Furthermore, if $u \in G$ is a fixed order-unit, then $S(G, u)=$ $S\left(G^{(c)}, u\right)$. Finally, if $G$ is a simple Riesz group, so is $G^{(c)}$.

Proof. The first two claims hold directly from Definition 1.2. Also, if $G$ is simple, so is $G^{(c)}$. Clearly, we can assume that $G$ is noncyclic by [8, Theorem 14.3], and then, as $(G, u)$ satisfies condition $(\mathrm{D})$, so does $\left(G^{(c)}, u\right)$. Moreover, $S\left(G^{(c)}, u\right)$ is a Choquet simplex by [8, Theorem 11.4]. Hence, $G^{(c)}$ is a Riesz group because of [11, Proposition 2.7].

For simple groups, Theorem 1.3 answers [8, Open Problem 2] in the case (a). In order to answer it in case (b) we apply the proof of Theorem 1.3 to the group $G$ endowed with the positive cone $\widetilde{G}^{+}=\left\{x \in G:(\exists n \in \mathbb{N})\left(2^{n} x \in G^{++}\right)\right\} \cup\{0\}$.

We will define now the basic building blocks to construct perforated direct limits.

Definition 1.4 Let $H$ be a partially ordered abelian group. We will say that $H$ is a quasi-simplicial group if $H^{(c)}$ is a simplicial group.

Observe that, aside from simplicial groups, this kind of groups are not directed, or are not interpolation groups. Now, we will prove the main result of this section:

Theorem 1.5 If $G$ is a directed torsion-free group such that $G^{(c)}$ is a dimension group, then there exists a direct system $\left(H_{i}, \widetilde{g}_{i}\right)_{i \in I}$ of quasi-simplicial groups such that $G=\lim _{\longrightarrow} H_{i}$.

Proof. To see this result, it suffices to prove that, if $G_{1}$ is a quasi-simplicial group, and $g_{1}: G_{1} \rightarrow G$ is a positive morphism, then there exist a quasi-simplicial group $G_{2}$, and there exist positive morphisms $h: G_{1} \rightarrow G_{2}$ and $g_{2}$ : $G_{2} \rightarrow G$ such that $g_{1}=g_{2} h$ and Ker $g_{1}=\operatorname{Ker} h$. If this assertion holds, then the rest of the proof is analogous to [8, Theorem 3.17] and [8, Theorem 3.19].

By the nature of $G_{1}$ and $G$, we can send the problem to $G_{1}{ }^{(c)}$ and $G^{(c)}$ throughout the identity morphism between partially ordered abelian groups and their canonical strictly unperforated images. Now, by $[8$, Proposition 
3.16], there exists a simplicial group $\widetilde{G_{2}}$ and there exist positive morphisms $\widetilde{g_{2}}: \widetilde{G}_{2} \rightarrow G^{(c)}$ and $\widetilde{h}: G_{1}^{(c)} \rightarrow \widetilde{G}_{2}$ such that $g_{1}=\widetilde{g_{2}} \widetilde{h}$ and Ker $g_{1}=\operatorname{Ker} \widetilde{h}$. Now, view $\widetilde{g_{2}}$ and $\widetilde{h}$ as homomorphisms of partially ordered abelian groups from $G_{1}$ to $\widetilde{G_{2}}$ and from $\widetilde{G_{2}}$ to $G$ respectively. Notice that $\widetilde{g_{2}}\left({\widetilde{G_{2}}}^{+}\right)$is a subcone of $G^{(c)+}$. Now, we endow $\widetilde{G_{2}}$ with the new positive cone $G_{2}{ }^{+}={\widetilde{G_{2}}}^{+} \cap{\widetilde{g_{2}}}^{-1}\left(G^{+}\right)$, that is a strict cone, and we verify that $G_{2}{ }^{(c)+}={\widetilde{G_{2}}}^{+}$. For all $x \in G_{2}^{(c)+}$ there exists $n \in \mathbb{N}$ such that $n x \in G_{2}{ }^{+}$, thus a fortiori $n x \in \widetilde{G}_{2}{ }^{+}$, and hence, since ${\widetilde{G_{2}}}^{+}$is a simplicial group, $x \in{\widetilde{G_{2}}}^{+}$. Conversely, if $x \in{\widetilde{G_{2}}}^{+}$, then $\widetilde{g_{2}}(x) \in G^{(c)+}$, i.e., there exists $n \in \mathbb{N}$ such that $n \widetilde{g_{2}}(x) \in G^{+}$(because $G$ is torsion-free), so that $n x \in{\widetilde{g_{2}}}^{-1}\left(G^{+}\right)$, but also $n x \in \widetilde{G}_{2}^{+}$, so that $n x \in G_{2}^{+}$. Hence $x \in G_{2}^{(c)+}$, and so we have proved that $G_{2}{ }^{(c)+}={\widetilde{G_{2}}}^{+}$. In particular, this means that $\widetilde{h}\left(G_{1}^{+}\right) \subseteq G_{2}^{+}$. Thus the assertion holds. So the result is proved in the same way of [8, Theorem 3.17, Corollary 3.18 and Theorem 3.19].

In particular, this result allows us to understand better the structure of torsion-free simple Riesz groups.

Corollary 1.6 Every torsion-free simple Riesz group is a direct limit of a direct system of quasi-simplicial groups.

Proof. Simply notice that, by Theorem 1.3, such a group satisfies the hypotheses of Theorem 1.5, whence the result holds.

\section{Additive subgroups of the rationals}

In this section we show some (probably well-known) results relating additive subgroups of $\mathbb{Q}$ and generalized integers.

From now on, let $\mathbb{P}$ denote the set of all primes. If $a$ and $b$ are integers, we write $a \mid b$ (respectively $a \perp b$ ) for " $a$ divides $b$ " (respectively "g.c.d. $(a, b)=1$ ").

Definition 2.1 A generalized integer $\mathfrak{n}$ is a map

$$
\mathfrak{n}: \mathbb{P} \longrightarrow\{0,1,2, \ldots, \infty\} .
$$

We denote the set of generalized integers by $\widetilde{\mathbb{N}}$. Sometimes we write

$$
\mathfrak{n}=\prod_{p \in \mathbb{P}} p^{\mathfrak{n}(p)}
$$

When $\mathfrak{n}$ is finite (i.e. it never takes the value $\infty$ and it is zero except at finitely many primes), we identify $\mathfrak{n}$ with the integer appearing on the right hand side of $(1)$. 
Notice that the notion of divisibility of integer numbers extends to the context of generalized integers: given $\mathfrak{m}, \mathfrak{n} \in \widetilde{\mathbb{N}}$, we say that $\mathfrak{n} \mid \mathfrak{m}$ if there exists $\mathfrak{n}^{\prime} \in \widetilde{\mathbb{N}}$ such that $\mathfrak{m}=\mathfrak{n} \cdot \mathfrak{n}^{\prime}$, that is, for each $p \in \mathbb{P}$ we have $\mathfrak{m}(p)=\mathfrak{n}(p)+\mathfrak{n}^{\prime}(p)$.

Lemma 2.2 Let $\mathfrak{n}$ be a generalized integer. Then:

(i) If $a, b \in \mathbb{N}$ and $a|\mathfrak{n}, b| \mathfrak{n}$, then l.c.m. $(a, b) \mid \mathfrak{n}$.

(ii) Let $\mathfrak{n}$ be infinite, and let $a \in \mathbb{N}$. If $a \mid \mathfrak{n}$, then $\mathfrak{n} / a$ is also infinite.

(iii) Let $\mathfrak{n}$ be infinite, and let $a \in \mathbb{N}$. Then there exists $b \in \mathbb{N}$ with $a \leq b$ such that $b \mid \mathfrak{n}$.

Proof. (i) It is obvious by the above remark.

(ii) As $\mathfrak{n}$ is infinite, $\sum_{p \in \mathbb{P}} \mathfrak{n}(p)=\infty$, while $\sum_{p \in \mathbb{P}} a(p)<\infty$ because $a \in \mathbb{N}$. So the result holds by definition.

(iii) Let $k$ be the least positive integer such that $a \leq 2^{k}$. Since $\mathfrak{n}$ is infinite, there exist positive integers $r, m_{1}, \ldots, m_{r}$ and there exist $p_{1}, \ldots, p_{r} \in \mathbb{P}$ such that $m_{i} \leq \mathfrak{n}\left(p_{i}\right)$ for all $i \in\{1, \ldots, r\}$ and $\sum_{i=1}^{r} m_{i}=k$. Put $b=\prod_{1<i<r} p_{i} m_{i}$, and notice that $b \mid \mathfrak{n}$. Since $p \geq 2$ for all $p \in \mathbb{P}$, we have $a \leq 2^{k} \leq b$, so we are done.

Lemma 2.3 Let $G$ be an additive subgroup of $\mathbb{Q}$ containing 1 , and let $a, b$ be natural numbers. Then:

(i) If $a \perp b$ and $a / b \in G$, then $1 / b \in G$.

(ii) If $b \mid a$ and $1 / a \in G$, then $1 / b \in G$.

(iii) If $1 / a, 1 / b \in G$, then $1 /$ l.c.m. $(a, b) \in G$.

Proof. (i) Since $a \perp b$, there exist $r, s \in \mathbb{Z}$ such that $a r+b s=1$. Then, as $1 \in G$, so is $r a / b+s=(a r) / b+(b s) / b=1 / b$, as desired.

(ii) There exists a natural number $c$ such that $a=b c$. Then, $1 / b=c / b c=$ $c / a \in G$.

(iii) There exist integers $r, s$ such that $r a+s b=$ g.c.d. $(a, b)$. Then, as $a \cdot b=$ l.c.m. $(a, b) \cdot$ g.c.d. $(a, b)$, we have that $1 /$ l.c.m. $(a, b)=$ g.c.d. $(a, b) / a b=$ $s / a+r / b \in G$.

Now, given $G$ an additive subgroup of $\mathbb{Q}$ containing 1 , we associate to $G$ a generalized integer $\mathfrak{n}_{G}$ in the following way: let $D_{G}=\{d \in \mathbb{N}: 1 / d \in G\}$, and define $\mathfrak{n}_{G}$ to be the least generalized integer such that $d \mid \mathfrak{n}_{G}$ for all $d \in D_{G}$. Notice that by Lemma 2.3(ii-iii)

$$
\mathfrak{n}_{G}=\prod_{p \in \mathbb{P} \cap D_{G}} p^{e(p)},
$$

where $e(p)$ is the supremum of $n \in \mathbb{N}$ such that $p^{n} \in D_{G}$. 
Similarly, given $\mathfrak{n}$ a generalized integer, we associate to $\mathfrak{n}$ an additive subgroup of $\mathbb{Q}$ containing 1 in the following way: put $\mathbb{Z}_{\mathfrak{n}}=\{a / b:(a \in \mathbb{Z})(b \mid \mathfrak{n})\}$. It is easy to see that $\mathbb{Z}_{\mathfrak{n}}$ is an additive subgroup of $\mathbb{Q}$ (by using Lemma $2.3($ ii-iii) $)$, and it contains 1 by definition.

We are ready to show a result that relates generalized integers and additive subgroups of $\mathbb{Q}$ containing 1 .

Lemma 2.4 (i) There is a one-to-one correspondence between $\widetilde{\mathbb{N}}$ and the set of additive subgroups of $\mathbb{Q}$ containing 1 .

(ii) Let $\mathfrak{n}$ be a generalized integer. Then, $\mathbb{Z}_{\mathfrak{n}}$ is a dense subgroup of $\mathbb{Q}$ if and only if $\mathfrak{n}$ is infinite.

Proof. (i) We will show that the maps defined above between generalized integers and additive subgroups of $\mathbb{Q}$ containing 1 are mutually inverse.

First, let $\mathfrak{n}$ be a generalized integer, and consider $\mathfrak{n}_{\mathbb{Z}_{\mathfrak{n}}}$. Fix $p \in \mathbb{P}$ and $a \in \mathbb{N}$. If $p^{a} \mid \mathfrak{n}$, then $1 / p^{a} \in \mathbb{Z}_{\mathfrak{n}}$ by definition of this group, and thus $p^{a} \mid \mathfrak{n}_{\mathbb{Z}_{\mathfrak{n}}}$ by definition of the generalized integer associated to $\mathbb{Z}_{\mathfrak{n}}$. Conversely, if $p^{a} \mid \mathfrak{n}_{\mathbb{Z}_{\mathfrak{n}}}$, then by definition of this integer and Lemma 2.3(ii) $1 / p^{a} \in \mathbb{Z}_{\mathfrak{n}}$, and thus $p^{a} \mid \mathfrak{n}$ by definition of $\mathbb{Z}_{\mathfrak{n}}$. Hence, $\mathfrak{n}=\mathfrak{n}_{\mathbb{Z}_{\mathfrak{n}}}$.

Now, let $G$ be an additive subgroup of $\mathbb{Q}$ containing 1 , and consider the group $\mathbb{Z}_{\mathfrak{n}_{G}}$. Fix $p / q \in \mathbb{Q}$ with $p \perp q$. If $p / q \in G$, then $1 / q \in G$ by Lemma 2.3(i), so that $q \mid \mathfrak{n}_{G}$, and thus $p / q \in \mathbb{Z}_{\mathfrak{n}_{G}}$. Conversely, if $p / q \in \mathbb{Z}_{\mathfrak{n}_{G}}$ with $p \perp q$, then $q \mid \mathfrak{n}_{G}$ by definition. Thus $1 / q \in G$ and so does $p / q$. Hence $G=\mathbb{Z}_{\mathfrak{n}_{G}}$.

(ii) It is well-known that every additive subgroup of $\mathbb{R}$ is either dense or cyclic. Notice that the cyclic subgroups of $\mathbb{R}$ containing 1 are those of the form $G=(1 / n) \mathbb{Z}$ for any $n \in \mathbb{N}$, and thus $\mathfrak{n}_{G}=n \in \mathbb{N}$ by part (i), whence the result holds.

\section{Construction of strictly perforated simple Riesz groups}

In this section, we give a procedure to construct examples of strictly perforated, torsion-free, simple Riesz groups. The idea is to use the fact that such a group is, according to Corollary 1.6, the direct limit of a direct system of quasi-simplicial groups. For our concrete construction, we will take as basic building blocks quasi-simplicial groups which are isomorphic to $\mathbb{Z}$ as abelian groups, and also that are directed and simple as partially ordered abelian groups. For the sake of simplicity we will call these groups simple components. Notice that the simple components are exactly the groups $G=\left(\mathbb{Z}, G^{+}\right)$, where $G^{+}=\left\{0, n_{1}, \cdots, n_{k}\right\} \cup\left(m+\mathbb{Z}^{+}\right)$for suitable $n_{1}<_{\mathbb{Z}} \cdots<_{\mathbb{Z}} n_{k}<_{\mathbb{Z}} m \in \mathbb{N}$ with $n_{k}+2 \leq_{\mathbb{Z}} m$ (in order to guarantee that $m$ is uniquely determined). Thus, 
our construction provides us with examples of rank one, while the examples of Lawrence [10] have infinite rank.

We start by showing some results of elementary number theory that will be useful in the sequel. To do this, we fix some notation. For every real number $x$, let $\lfloor x\rfloor$ (respectively $\lceil x\rceil$ ) denote the largest integer $\leq x$ (respectively the least integer $\geq x)$.

Lemma 3.1 Let $I$ be an interval of $\mathbb{R}$, let $\ell$ be the length of $I$ and let $m \in \mathbb{N}$. Then we have

$$
\frac{\ell}{m}-1<|I \cap m \mathbb{Z}| \leq \frac{\ell}{m}+1 \text {. }
$$

Proof. Put $I=[a, b]$ (so that $\ell=b-a$ ). For all $x \in \mathbb{Z}, m x$ belongs to $I$ if and only if

$$
\lceil a / m\rceil \leq x \leq\lfloor b / m\rfloor,
$$

so that the desired number is $|I \cap m \mathbb{Z}|=\lfloor b / m\rfloor-\lceil a / m\rceil+1$. But we have $b / m-1<\lfloor b / m\rfloor \leq b / m$ and $a / m \leq\lceil a / m\rceil<a / m+1$, so that it follows that

$$
(b / m-1)-(a / m+1)+1<|I \cap m \mathbb{Z}| \leq b / m-a / m+1
$$

and the conclusion follows.

Lemma 3.2 Let $\left\langle p_{i}: i<k\right\rangle$ be a (finite) list of mutually distinct primes, let $I$ be an interval of $\mathbb{R}$ and let $\ell$ be the length of $I$. Put

$$
X=\left\{x \in I \cap \mathbb{Z}:(\forall i<k)\left(p_{i} \perp x\right)\right\} .
$$

Then we have

$$
|X| \geq \ell \cdot \prod_{i<k}\left(1-1 / p_{i}\right)-2^{k} .
$$

Proof. For all $i<k$, put $X_{i}=I \cap p_{i} \mathbb{Z}$. Then we have $X=(I \cap \mathbb{Z}) \backslash \bigcup_{i<k} X_{i}$, thus, by the sieve formula,

$$
|X|=\sum_{0 \leq n \leq k}\left[(-1)^{n} \cdot \sum_{U \in[k]^{n}}\left|X_{U}\right|\right]
$$

where we define $[k]^{n}$ as the set of all $n$-elements subsets of $\{0,1, \ldots, k-1\}$ and we put $X_{U}=\bigcap_{i \in U} X_{i}$ (and $X_{\emptyset}=I \cap \mathbb{Z}$ ). However, if we put $q_{U}=\prod_{i \in U} p_{i}$ (and $\left.q_{\emptyset}=1\right)$, we have

$$
X_{U}=\left\{x \in I \cap \mathbb{Z}: q_{U} \mid x\right\},
$$

thus by Lemma 3.1, $\ell / q_{U}-1<\left|X_{U}\right| \leq \ell / q_{U}+1$. It follows that $|X| \geq \ell \alpha-\beta$, where we put

$$
\alpha=\sum_{0 \leq n \leq k}\left[(-1)^{n} \cdot \sum_{U \in[k]^{n}} 1 / q_{U}\right]=\prod_{i<k}\left(1-1 / p_{i}\right)
$$


and

$$
\beta=\sum_{0 \leq n \leq k}\left(\begin{array}{c}
k \\
n
\end{array}\right)=2^{k} .
$$

Lemma 3.3 (i) Let $I$ be an interval of $\mathbb{R}$ of length $\ell \geq 2$, and let $p \in \mathbb{P}$. Then $I \cap \mathbb{Z}$ contains an element coprime with $p$.

(ii) Let $I$ be an interval of $\mathbb{R}$ of length $\ell \geq 4$ and let $p, q \in \mathbb{P}$. Then $I \cap \mathbb{Z}$ contains an element coprime with both $p$ and $q$.

Proof. (i) Apply Lemma 3.1 with $m=1$. Then $1 \leq \ell-1<|I \cap \mathbb{Z}|$, so there are at least two consecutive integers in $I$. One of them must be necessarily coprime with $p$, as otherwise $p \mid 1$, a contradiction.

(ii) By the result of (i), one can suppose that $p \neq q$. Again, apply Lemma 3.1 with $m=1$. Then $3 \leq \ell-1<|I \cap \mathbb{Z}|$, so there are at least four consecutive integers $n, n+1, n+2, n+3$ in $I \cap \mathbb{Z}$. Suppose that the property fails for $I$. Hence, without loss of generality $p \mid n$. One cannot have $p \mid n+1$, so that $q \mid n+1$. Similarly $p \mid n+2$ and $q \mid n+3$. Therefore, $p \mid 2$ and $q \mid 2$, a contradiction.

From now on, let $\varphi$ be the Euler function, let $\nu: \mathbb{N} \longrightarrow \mathbb{Z}^{+}$be the function defined by the rule $\nu(n)=$ number of prime factors of $n$. Define a function $\tau: \mathbb{N} \longrightarrow \mathbb{Q}^{+}$by putting

$$
\tau(n)=\frac{2^{\nu(n)}}{\varphi(n)} .
$$

Proposition 3.4 Let $n \geq 2$ be an integer and let $I$ be an interval of $\mathbb{R}$. If the length of $I$ is $>\tau(n) n$, then $I \cap \mathbb{Z}$ contains an element coprime with $n$.

Proof. Let $n=\prod_{i<k} p_{i}^{e_{i}}$ be the decomposition of $n$ into prime factors (so that $k=\nu(n)$, the $p_{i}{ }^{\prime}$ 's are mutually distinct primes and the $e_{i}$ 's belong to $\mathbb{N}$ ), and let $\ell$ be the length of $I$. Then the number of elements of $I \cap \mathbb{Z}$ which are coprime with $n$ is, by Lemma 3.2 , at least equal to $\ell \cdot \prod_{i<k}\left(1-1 / p_{i}\right)-2^{k}=$ $\ell \cdot \varphi(n) / n-2^{k}>\left(2^{k} / \varphi(n)\right) \cdot n \cdot(\varphi(n) / n)-2^{k}=0$.

Corollary 3.5 Let $n \geq 3$ be an integer and let $I$ be an interval of $\mathbb{R}$. If the length of $I$ is $>\frac{2}{3} n$, then $I \cap \mathbb{Z}$ contains an element coprime with $n$.

Proof. Let again $\ell$ be the length of $I$. If $n \in\{3,4,5\}$ then $\ell \geq 2$ and we conclude by Lemma 3.3(i). If $5<n<2 \cdot 3 \cdot 5=30$ then $\nu(n)=2$ and $\ell>2 / 3 \cdot n \geq 2 / 3 \cdot 6=4$, whence we conclude again by Lemma 3.3(ii). If $n=30$, then $\ell \geq 20$, thus there exists an even integer $m \in I$ such that $m+5 \in I$. If no element of $I \cap \mathbb{Z}$ is coprime with 30, then every element of 
the set $J=\{m+1, m+3, m+5\}$ would be a multiple of either 3 or 5 . By the Pigeonhole Principle, either 3 or 5 divides two distinct elements of $J$. Then, in any case, either 3 or 5 divides the difference between two elements of $J$, i.e., 3 or 5 divides 2 or 4 , a contradiction.

Thus let us suppose from now on that $n \geq 31$. To conclude the desired result, it suffices by Proposition 3.4 to prove that $\tau(n) \leq 2 / 3$. If $\nu(n) \leq 2$, as $\ell>2 / 3 \cdot n \geq 20>4$, we conclude again by Lemma 3.3(ii), so we can suppose from now on that $\nu(n) \geq 3$. If 2,3 and 5 are the only prime factors of $n$, then there are $a, b, c \in \mathbb{N}$ (with at least one of them $\geq 2$, since $n>30$ ) such that $n=2^{a} 3^{b} 5^{c}$ and thus

$$
\tau(n)=\frac{2}{2^{a-1}} \cdot \frac{2}{3^{b-1} \cdot 2} \cdot \frac{2}{5^{c-1} \cdot 4}=\frac{1}{2^{a-1} \cdot 3^{b-1} \cdot 5^{c-1}},
$$

whence $\tau(n) \leq 1 / 2<2 / 3$. The only remaining possibility is when $n$ admits a prime factor $p \geq 7$. Then, if $n=\prod_{i<k} p_{i}^{e_{i}}$, we have

$$
\tau(n)=\prod_{i<k} \frac{2}{p_{i}^{e_{i}-1}\left(p_{i}-1\right)} .
$$

Thus, if $p_{i} \geq 3$ then $2 / p_{i}^{e_{i}-1}\left(p_{i}-1\right) \leq 1$, and if $p_{i}=2$ then $2 / p_{i}^{e_{i}-1}\left(p_{i}-1\right) \leq 2$. Hence by (2)

$$
\tau(n) \leq 2 \cdot \frac{2}{p-1} \leq 4 / 6=2 / 3
$$

Now we introduce a concept that will be useful in the sequel. If $G$ is a partially ordered abelian group, we say that an interpolation problem of $G$ is a quadruple of the form $\alpha=\left(x_{1}, x_{2}, y_{1}, y_{2}\right)$ of elements of $G$ satisfying $\left\{x_{1}, x_{2}\right\} \leq_{G}\left\{y_{1}, y_{2}\right\}$, while a solution of $\alpha$ is an element $z \in G$ satisfying $\left\{x_{1}, x_{2}\right\} \leq_{G} z \leq_{G}\left\{y_{1}, y_{2}\right\}$. If $G$ is a simple component, we say that a reduced interpolation problem is an interpolation problem of the form $\left(0, x, y_{1}, y_{2}\right)$ with $0<_{\mathbb{Z}} x<_{\mathbb{Z}} y_{1}<_{\mathbb{Z}} y_{2}$. The following results guarantees that, with this kind of building blocks, we can construct a direct limit that in each step "interpolates" the elements of the previous step without losing the perforation property. Given $G=\left(\mathbb{Z}, G^{+}\right)$a simple component with $G^{+}=\left\{0, n_{1}, \ldots, n_{k}\right\} \cup\left(m+\mathbb{Z}^{+}\right)$, we denote $q_{G}=16 \mathrm{~m}$.

Lemma 3.6 Let $G=\left(\mathbb{Z}, G^{+}\right)$be a simple component, and let $\alpha$ be a reduced interpolation problem of $G$. Then for all $q \geq q_{G}$ there exists a simple component $H$ such that $q$. (i.e., multiplication by q) defines an order-embedding of partially ordered abelian groups from $G$ into $H$ such that $q \alpha$ admits a solution in $H$.

Proof. As we noticed above, such a group has the form $G=\mathbb{Z}, G^{+}=$ $\left\{0, n_{1}, \ldots, n_{k}\right\} \cup\left(m+\mathbb{Z}^{+}\right)$for suitable $n_{1}<_{\mathbb{Z}} \cdots<_{\mathbb{Z}} n_{k}<_{\mathbb{Z}} m$ of $\mathbb{N}$. Set $\alpha=\left(0, x, y_{1}, y_{2}\right)$ the reduced interpolation problem. 
Let $q \in \mathbb{N}$ such that $q \geq q_{G}=16 \mathrm{~m}$. Then, the interval $I=[q(x+$ $\left.1 / 8), q\left(y_{1}-1 / 8\right)\right]$ of $\mathbb{Q}$ has length $q\left(y_{1}-x-1 / 4\right) \geq 3 / 4 \cdot q>2 / 3 \cdot q$. Thus by Corollary 3.5 there exists $r \in I \cap \mathbb{Z}$ coprime with $q$.

Let $S$ be the submonoid of $\mathbb{Z}^{+}$generated by $\left\{r, r-q x, q y_{1}-r, q y_{2}-r\right\} \cup q G^{+}$, and let $H$ be the simple component defined by $H=\mathbb{Z}, H^{+}=S \cup\left(q m+\mathbb{Z}^{+}\right)$. Let $g: G \rightarrow H$ be the morphism defined by multiplication by $q$, that is clearly positive because of the definition of $S$. Also notice that $\{0, q x\} \leq_{H} r \leq_{H}$ $\left\{q y_{1}, q y_{2}\right\}$, i.e., that $r$ is a solution of the problem $q \alpha$. It remains to show that $g$ is an order-embedding. Thus, let $t \in G$ such that $g(t) \in H^{+}$, and notice that $t \in \mathbb{Z}^{+}$. If $t \geq_{\mathbb{Z}} m$, then $t \in G^{+}$directly. So suppose that $0<_{\mathbb{Z}} t<_{\mathbb{Z}} m$. It follows that $g(t) \in S$, and thus there are $\alpha_{1}, \alpha_{2}, \beta_{1}, \beta_{2}$ in $\mathbb{Z}^{+}$and $h$ in $G^{+}$such that $q t=\alpha_{1} r+\alpha_{2}(r-q x)+\beta_{1}\left(q y_{1}-r\right)+\beta_{2}\left(q y_{2}-r\right)+q h$; dividing by $q$ yields

$$
t=\alpha_{1} r / q+\alpha_{2}(r-q x) / q+\beta_{1}\left(q y_{1}-r\right) / q+\beta_{2}\left(q y_{2}-r\right) / q+h .
$$

By definition of $r$, the numbers $r / q,(r-q x) / q,\left(q y_{1}-r\right) / q$ and $\left(q y_{2}-r\right) / q$ are larger or equal than $1 / 8$. Thus it follows that if one of the $\alpha_{i}$ 's or the $\beta_{i}$ 's is larger or equal than $8 m$, then $t \geq_{\mathbb{Z}} m$, a contradiction. Therefore, if we define $\delta=\left(\alpha_{1}+\alpha_{2}\right)-\left(\beta_{1}+\beta_{2}\right)$, it satisfies $-16 m<_{\mathbb{Z}} \delta<_{\mathbb{Z}} 16 m$, thus a fortiori

$$
-q<_{\mathbb{Z}} \delta<_{\mathbb{Z}} q
$$

On the other hand, rearranging (3) yields

$$
t=\delta r / q+\left(\beta_{1} y_{1}+\beta_{2} y_{2}-\alpha_{2} x\right)+h,
$$

whence $q \mid \delta \cdot r$, but also we have that $r \perp q$, and thus $q \mid \delta$. Hence, by (4), $\delta=0$, i.e., $\alpha_{1}+\alpha_{2}=\beta_{1}+\beta_{2}$. Now, by Riesz decomposition in $\mathbb{Z}^{+}$, there are $\gamma_{i} \leq \beta_{i}(i \in\{1,2\})$ in $\mathbb{Z}^{+}$such that $\alpha_{2}=\gamma_{1}+\gamma_{2}$. Therefore we obtain that

$$
t=\left(\beta_{1}-\gamma_{1}\right) y_{1}+\gamma_{1}\left(y_{1}-x\right)+\left(\beta_{2}-\gamma_{2}\right) y_{2}+\gamma_{2}\left(y_{2}-x\right)+h \in G^{+},
$$

so we are done.

Lemma 3.7 Let $G=\left(\mathbb{Z}, G^{+}\right)$be a simple component. Then:

(i) For each interpolation problem $\alpha$ of $G$ there exists a reduced interpolation problem $\alpha^{\prime}$ of $G$ and there exists an element $n \in G$ such that if $r \in G$ is a solution of $\alpha^{\prime}$ then $r+n \in G$ is a solution of $\alpha$.

(ii) There are only finitely many reduced interpolation problems of $G$ without solution in $G$.

Proof. (i) Let $\alpha=\left(x_{1}, x_{2}, y_{1}, y_{2}\right)$ be an interpolation problem of $G$. We can assume that these elements, as elements of $\mathbb{Z}$ with the natural ordering, are ordered as follows:

$$
x_{1} \leq_{\mathbb{Z}} x_{2} \leq_{\mathbb{Z}} y_{1} \leq_{\mathbb{Z}} y_{2} .
$$


If $x_{1}=x_{2}$, or $x_{2}=y_{1}$, or $y_{1}=y_{2}$, then $\alpha$ admits a trivial solution in $G$. So that we can assume that

$$
x_{1}<_{\mathbb{Z}} x_{2}<_{\mathbb{Z}} y_{1}<_{\mathbb{Z}} y_{2} .
$$

Put $x=x_{2}-x_{1}, \bar{y}_{1}=y_{1}-x_{1}$ and $\bar{y}_{2}=y_{2}-x_{1}$. Thus we have $\alpha^{\prime}=\left(0, x, \bar{y}_{1}, \bar{y}_{2}\right)$ a reduced interpolation problem. If $r$ is a solution for $\alpha$, so is $r^{\prime}=r-x_{1}$ for $\alpha^{\prime}$, and conversely, if $r^{\prime}$ is a solution for $\alpha^{\prime}$, so is $r=r^{\prime}+x_{1}$ for $\alpha$.

(ii) Let $\alpha=\left(0, x, y_{1}, y_{2}\right)$ be a reduced interpolation problem in $G$, where $G^{+}=\left\{0, n_{1}, \ldots, n_{k}\right\} \cup\left(m+\mathbb{Z}^{+}\right)$for suitable $n_{1}<_{\mathbb{Z}} \cdots<_{\mathbb{Z}} n_{k}<_{\mathbb{Z}} m$ of $\mathbb{N}$. If $x \in G^{+}$or $y_{2}-y_{1} \in G^{+}$, then the interpolation problem admits a trivial solution. Thus, the only possibilities for $\alpha$ having no solution in $G$ are $0<_{\mathbb{Z}} x<_{\mathbb{Z}} m$ and $x \notin G^{+}, y_{1}<_{\mathbb{Z}} 2 m+x$ and $y_{1}<_{\mathbb{Z}} y_{2}<_{\mathbb{Z}} m+y_{1}$, but $y_{2}-y_{1} \notin G^{+}$. In particular, $\left\{x, y_{1}, y_{2}\right\}<_{\mathbb{Z}} 4 m$, which ends the proof.

Definition 3.8 (a) For every generalized integer $\mathfrak{n}$, a sequence $\left\{a_{n}\right\}_{n \geq 1}$ of positive integers is associated to $\mathfrak{n}$ when $\mathfrak{n}=\prod_{n \geq 1} a_{n}$.

(b) For every simple component $G$ and every finite list of interpolation problems $\alpha_{1}, \cdots, \alpha_{t}$ of $G$, let $E\left(G ; \alpha_{1}, \cdots, \alpha_{t}\right)$ be the set of all positive integers $q$ such that there exists a simple component $H$ satisfying both following conditions:

(i) The multiplication map $q \cdot$ defines an embedding of ordered groups from $G$ into $H$.

(ii) For all $i \in\{1, \ldots, t\}, q \alpha_{i}$ admits a solution in $H$.

If $\alpha_{1}, \cdots, \alpha_{t}$ is the list of all reduced interpolation problems of $G$ without a solution in $G$ (which is finite because of Lemma 3.7), we write $E(G)$ instead of $E\left(G ; \alpha_{1}, \cdots, \alpha_{t}\right)$.

As a consequence of these lemmas we have the following result.

Lemma 3.9 Let $G=\left(\mathbb{Z}, G^{+}\right)$be a simple component, let $\mathfrak{n}$ be an infinite generalized integer, let $\left\{a_{n}\right\}_{n \geq 1}$ be a sequence associated to $\mathfrak{n}$. Then there are arbitrary large $k \in \mathbb{N}$ such that $q_{k}=a_{1} \cdots a_{k}$ belongs to $E(G)$.

Proof. Let $E(G)=\left\{\alpha_{1}, \ldots, \alpha_{t}\right\}$ be the set of reduced interpolation problems of $G$ without solution in $G$. We will prove the result by induction on $t$. First suppose that $t=1$. Since $\mathfrak{n}$ is infinite, there exists $k \in \mathbb{N}$ such that $q_{G} \leq_{\mathbb{Z}}$ $a_{1} \cdots a_{n}$ for all $n \geq k$, whence the result holds because of Lemma 3.6. By induction hypothesis there are arbitrary large $k^{\prime} \in \mathbb{N}$ such that $q_{k^{\prime}}=a_{1} \cdots a_{k^{\prime}}$ belongs to $E\left(G ; \alpha_{1}, \ldots, \alpha_{t-1}\right)$. Fix one of these positive integers $k^{\prime}$, and notice that there exists a simple component $H^{\prime}$ such that the map $q_{k^{\prime}}$. defines an embedding of ordered groups from $G$ into $H^{\prime}$, and for all $i \in\{1, \ldots, t-1\}$, $q \alpha_{i}$ admits a solution in $H^{\prime}$. As $\mathfrak{n}$ is infinite, by Lemma 2.2(ii) there exists an 
infinite generalized integer $\mathfrak{n}^{\prime}$ such that $\mathfrak{n}=q_{k^{\prime}} \cdot \mathfrak{n}^{\prime}$. Now, the sequence $\left\{a_{k+i}\right\}_{i \geq 1}$ is associated to $\mathfrak{n}^{\prime}$, and again there exists $k^{\prime \prime}>k^{\prime}$ such that $q_{H^{\prime}} \leq{ }_{\mathbb{Z}} a_{k^{\prime}+1} \cdots a_{n}$ for all $n \geq k^{\prime \prime}$. Hence by Lemma 3.6, for each $n \geq k^{\prime \prime}$ there exists a simple component $H_{n}$ such that the multiplication map $q_{n}^{\prime}$. (where $q_{n}^{\prime}=a_{k^{\prime}+1} \cdots a_{n}$ ) defines an embedding of ordered groups from $H^{\prime}$ into $H_{n}$, and for all $i \in$ $\{1, \ldots, t\}, q_{n}^{\prime} q_{k^{\prime}} \alpha_{i}$ admits a solution in $H_{n}$. Thus for each $n \geq k^{\prime \prime}$ the positive integer $q_{n}=a_{1} \cdots a_{n}$ belongs to $E(G)$, and hence the induction step holds, which ends the proof.

Lemma 3.10 Let $\mathfrak{n}$ be an infinite generalized integer. Then:

(i) There exists a sequence associated to $\mathfrak{n}$.

(ii) Let $\left\{a_{n}\right\}_{n \geq 1}$ be a sequence associated to $\mathfrak{n}$, let $\left\{G_{n}\right\}_{n \geq 1}$ be a sequence of simple components such that for all $n \geq 1, g=a_{n}$. is an embedding of ordered groups from $G_{n}$ into $G_{n+1}$. For all $n \in \mathbb{N}$, let $h_{n}: G_{n} \rightarrow \mathbb{Z}_{\mathfrak{n}}$ be defined by the rule $h_{n}(x)=\left(1 / a_{1} \cdots a_{n}\right) x$. Put $G=\mathbb{Z}_{\mathfrak{n}}, G^{+}=\bigcup_{n \geq 1} h_{n}\left(G_{n}^{+}\right)$. Then $\left(G, G^{+}\right)$is the direct limit of the direct system $\left(G_{n}, G_{n}^{+}, g_{n}\right)$ with limiting maps $h_{n}: G_{n} \rightarrow G$.

Proof. (i) For each $n \geq 1$ and for each $p \in \mathbb{P}$ with $p \leq n$, define $\varphi(n, p)=$ $\min \{n, \mathfrak{n}(p)\}$. Now, let $\left\{t_{n}\right\}_{n \geq 1}$ be the sequence with terms

$$
t_{n}=\prod_{p \leq n, p \in \mathbb{P}} p^{\varphi(n, p)} .
$$

So we have that $t_{1}=1$, that $t_{n} \mid t_{m}$ for $1 \leq n \leq m$, and that $t_{n} \mid \mathfrak{n}$ for every $n \geq 1$. For $n \geq 1$, put $a_{n}=t_{n+1} / t_{n}$, and notice that for each $n \geq 1$ we have

$$
\prod_{i=1}^{n}=a_{1} \cdots a_{n}=t_{2} / t_{1} \cdots t_{n+1} / t_{n}=t_{n+1} .
$$

Obviously, if $p \in \mathbb{P}, n \geq 1$ and $p^{n} \mid t_{m}$ for some $m \geq 1$, then $p^{n} \mid \mathfrak{n}$. Conversely, if $p \in \mathbb{P}, n \geq 1$ and $p^{n} \mid \mathfrak{n}$, then $n \leq \mathfrak{n}(p)$, whence for any $m \geq \max \{n, p\}$ we have $p \leq m$ and $n \leq \varphi(n, p)$. Hence $p^{n} \mid t_{m}$, and thus

$$
\mathfrak{n}=\prod_{n \geq 1} a_{n}
$$

Consequently, $\left\{a_{n}\right\}_{n \geq 1}$ is a sequence associated to $\mathfrak{n}$, as desired.

(ii) The maps $g_{n}$ and $h_{n}$ satisfy the following properties: (a) For every $n \geq 1$ the morphism $h_{n}: G_{n} \rightarrow \mathbb{Z}_{\mathfrak{n}}$ is injective; (b) For every $n \geq 1$ the following diagram is commutative:

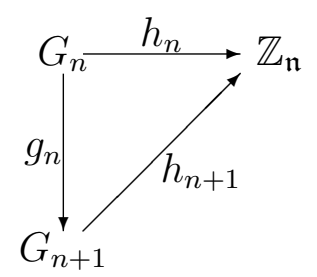


If we denote $H=\lim G_{n}$ and $\varphi_{n}: G_{n} \rightarrow H$ are the natural maps given by the direct limit construction then, by the Universal Property of direct limits, there is a unique group morphism $g: H \longrightarrow \mathbb{Z}_{\mathfrak{n}}$ (which is injective, as so is $h_{n}$ for all $n \geq 1$ ) that for every $n \geq 1$ satisfies

$$
g \varphi_{n}=h_{n}
$$

So, if $1 / q \in \mathbb{Z}_{\mathfrak{n}}$, then $q \mid \mathfrak{n}$, and as $\left\{a_{n}\right\}_{n \geq 1}$ is associated to $\mathfrak{n}$, there exist $m, k \in \mathbb{N}$ such that $q \cdot m=\prod_{1 \leq i \leq k} a_{i}$. By (6), if we take $m \in G_{k+1}$, then $g\left(\varphi_{k+1}(m)\right)=m /\left(a_{1} \cdots a_{k}\right)=1 / q$, whence the map $g$ is an isomorphism. Hence, $G$ is the direct limit of the given direct system with respect to the limiting maps $h_{n}: G_{n} \rightarrow G$, as desired.

As a consequence we obtain the following result.

Theorem 3.11 For every simple component $(\mathbb{Z}, M)$, and for every dense additive subgroup $G$ of $\mathbb{Q}$ containing $\mathbb{Z}$, there exists a submonoid $P$ of $G \cap \mathbb{Q}^{+}$ such that $G=P+(-P)$ and $(G, P)$ is a simple Riesz group, with $M=P \cap \mathbb{Z}^{+}$.

Proof. By Lemma 2.4(i-ii), $G=\mathbb{Z}_{\mathfrak{n}_{G}}$, where $\mathfrak{n}_{G}$ is an infinite generalized integer. Let $\left\{a_{n}\right\}_{n \geq 1}$ be the sequence defined in Lemma 3.10(i).

Now let $\left(G_{1}, G_{1}^{+}\right)=(\mathbb{Z}, M)$ be the given simple component. By Lemma 3.9 there exists $k_{1} \in \mathbb{N}$ such that $q_{1}=a_{1} \cdots a_{k_{1}}$ belongs to $E\left(G_{1}\right)$, with witness a simple component $G_{2}$. Let $\mathfrak{n}_{1}=\mathfrak{n} / q_{1}$, and apply Lemma 3.9 to the simple component $G_{2}$ and the infinite generalized integer $\mathfrak{n}_{1}$. Then there exists $k_{2}>k_{1}$ such that $q_{2}=a_{k_{1}+1} \cdots a_{k_{2}}$ belongs to $E\left(G_{2}\right)$, with witness a simple component $G_{3}$. So by recurrence we construct simple components $\left(G_{n}, G_{n}^{+}\right)$and group morphisms $g_{n}=q_{n} \cdot: G_{n} \rightarrow G_{n+1}$ satisfying: (a) For every $n \geq 1$ the map $g_{n}$ is an embedding of ordered groups from $G_{n}$ into $G_{n+1}$; (b) For every $n \geq 1$, if $\alpha$ is an interpolation problem in $G_{n}$, then $g_{n}(\alpha)$ admits a solution in $G_{n+1}$ (because of Lemma 3.7(i) and the fact that $q_{n}$ belongs to $E\left(G_{n}\right)$ with witness the group $\left.G_{n+1}\right)$.

We define maps $h_{n}: G_{n} \rightarrow \mathbb{Z}_{\mathfrak{n}_{G}}$ by the rule $h_{n}(x)=\left(1 / q_{1} \cdots q_{n-1}\right) x$, and we take $P=\bigcup_{n \geq 1} h_{n}\left(G_{n}^{+}\right)$. Then, since $\left\{q_{n}\right\}_{n \geq 1}$ is a sequence associated to $\mathfrak{n}$ (because of $(5))$, we have that $(G, P)$ is the direct limit of the direct system $\left(G_{n}, G_{n}^{+}, g_{n}\right)$ with limiting maps $h_{n}$ because of Lemma 3.10(ii). As each group $\left(G_{i}, G_{i}^{+}\right)$is simple and directed (because they are simple components), then so is $(G, P)$. Moreover, since each group morphism $g_{n}$ is an order-embedding (because of (a)), we have that $(G, P)$ is an interpolation group by (b). Finally, as $h_{1}(x)=x$, we have that $h_{1}(M)=M$, whence $P \cap \mathbb{Z}^{+}=M$, so we are done.

To end this note, we construct some examples of torsion-free, strictly perforated, simple Riesz groups that illustrate the construction technique described 
in Theorem 3.11. First of all, we fix the simple component $G_{1}=\left(\mathbb{Z}, G_{1}^{+}\right)$, where $G_{1}^{+}=\{0\} \cup\left(2+\mathbb{Z}^{+}\right)$. Now, according to Lemma 2.4(i) and Theorem 3.11 , for each infinite generalized integer that we fix, there is a dense additive subgroup $G$ of $\mathbb{Q}$ containing 1 and a strict cone $P$ such that $P \cap \mathbb{Z}^{+}=G_{1}^{+}$and $(G, P)$ is a simple Riesz group. So to obtain two examples, we fix (a) $\mathfrak{m}=2^{\infty}$, (b) $\mathfrak{n}=\prod_{p \in \mathbb{P}} p^{\infty}$ (where the primes appear in the natural ordering of $\mathbb{N}$ ). In case (a) the group that we obtain through the construction is $G=\mathbb{Z}[1 / 2]$, with associated sequence $\left\{a_{n}\right\}_{n \geq 1}=\left\{2,4,8, \ldots, 2^{n}, \ldots\right\}$, and in case (b) the group is $G=\mathbb{Q}$, with associated sequence $\left\{a_{n}\right\}_{n \geq 1}=\{4,54,6,18750, \ldots\}$. To apply the construction technique described in Lemmas 3.6 and 3.9, we need also to state which are the reduced interpolation problems in $G_{1}$ with no solution. According to Lemma 3.7, those are:

$$
\begin{aligned}
& \alpha_{1}=(0,1,3,4), \\
& \alpha_{2}=(0,1,4,5) \text {. }
\end{aligned}
$$

Then, in the first step of case (a) we have $q_{1}=2 \cdot 2^{2} \cdot 2^{3}=64$ and so if we take $r=73$ (which is coprime with 64 ) we obtain

$$
\begin{gathered}
G_{2}^{+}=\{0,9,18,27,36,45,54,63,72,73,81,82, \\
90,91,99,100,109,110,118,119\} \cup\left(127+\mathbb{Z}^{+}\right) .
\end{gathered}
$$

Similarly, in the first step of case (b) we have $q_{1}=4 \cdot 54=216$ and so if we take $r=245$ (which is coprime with 120) we obtain

$$
\begin{aligned}
& G_{2}^{+}=\{0,29,58,87,116,145,174,203,232,245,261,274,290, \\
& 303,319,334,348,361,377,390,403,406,419\} \cup\left(432+\mathbb{Z}^{+}\right) .
\end{aligned}
$$

In both cases $h_{2}\left(G_{2}^{+}\right) \cap \mathbb{Z}=G_{1}^{+}$. The examples are now obtained by applying recursively this procedure.

\section{Acknowledgments}

I thank P. Ara for his guidance and his support, K. R. Goodearl for his advice and a lot of interesting suggestions, and also the referee, whose reports have really improved the original version of this note.

\section{References}

[1] P. Ara, K. R. GoodearL, The almost isomorphism relation for simple regular rings, Pub. Mat. UAB, 36(2) (1992), pp. 369-388. 
[2] B. BlackAdAR, "K-Theory for operator algebras", M.S.R.I. Publ., 5, Springer-Verlag, New York, 1986.

[3] B. Blackadar, Comparison theory for simple $C^{*}$-algebras, "Operator algebras and application", D. E. Evans and M. Takesaki (eds.), LMS Lecture Notes Series, 135, Cambridge Univ. Press, 1988, pp. 21-54.

[4] M. Dugas, Unpublished example, 1994.

[5] E. G. Effros, D. E. Handelman and C.-L. Shen, Dimension groups and their affine representations, Amer. J. Math., 102 (1980), pp. 385-407.

[6] G. A. ElLiott, On the classification of inductive limits of sequences of semisimple finite-dimensional algebras, J. Algebra, 38 (1976), pp. 29-44.

[7] K. R. Goodearl, "von Neumann Regular Rings", Pitman, London, 1979; "," Second Ed., Krieger, Malabar, Fl., 1991.

[8] K. R. Goodearl, "Partially Ordered Abelian Groups with Interpolation", Math. Surveys and Monographs, 20, A.M.S., Providence, 1986.

[9] K. R. Goodearl, Torsion in $K_{0}$ of unit-regular rings, Proc. Edinburg Math. Soc., 38 (1995), pp. 331-341.

[10] J. W. LAWRENCE, Unpublished examples, 1981.

[11] E. PARDo, On a density condition for $K_{0}{ }^{+}$of von Neumann regular rings, Comm. Algebra, 22(2) (1994), pp. 707-719.

[12] E. PARdo, Metric completions of ordered groups and $K_{0}$ of exchange rings, Trans. Amer. Math. Soc. (to appear).

[13] F. Wehrung, Metric properties of positively ordered monoids, Forum Mathematicum, 5 (1993), pp. 183-201. 